\title{
Transformaciones socioeconómicas, migración y organización etnopolítica rural-urbana entre la Región Chaqueña y la Región Metropolitana de Buenos Aires
}

\section{Malena Castilla}

Universidad de Buenos Aires, Ciudad A. de Buenos Aires, Argentina

Correo Electrónico: malenacastilla@gmail.com

\section{María Laura Weiss}

Universidad de Buenos Aires, Instituto de Ciencias Antropológicas, Ciudad A. de Buenos Aires, Argentina CONICET, Ciudad A. de Buenos Aires, Argentina

Correo Electrónico: weissmlaura@gmail.com

\section{Juan Manuel Engelman}

Universidad de Buenos Aires, Instituto de Ciencias Antropológicas, Ciudad A. de Buenos Aires, Argentina CONICET, Ciudad A. de Buenos Aires, Argentina

Correo Electrónico: jmengelman@hotmail.com
Recibido

octubre de 2018

Aceptado

abril de 2019

doi: $10.34096 /$ cas.i49.5272

\section{Resumen}

El presente trabajo tiene por objetivo analizar el renovado proceso de expansión de la frontera agropecuaria y la constante migración de población indígena hacia diversos centros urbanos como la Región Metropolitana de Buenos Aires. Nos proponemos indagar acerca del impacto que poseen las grandes inversiones agropecuarias más recientes en torno a los procesos de urbanización y concentración de mano de obra indígena. Se trata de enseñar que, más allá del impacto negativo de la expropiación territorial, el quiebre de las economías domésticas y los circuitos de trabajo del siglo $\mathrm{XX}$, las poblaciones indígenas han reorientado sus desplazamientos migratorios en torno a nuevos formatos regionales basados en la lucha por el territorio, la identidad y el acceso a mejores condiciones de vida.

\section{Socioeconomic transformations, migration and rural-urban ethnopolitical organization between the Chaco Region and the Buenos Aires Metropolitan Region}

\section{Abstract}

This article explores the renewed process of expansion of the agricultural frontier, and the constant migration of indigenous population to different urban centers like the Metropolitan Region of Buenos Aires. In order to do so, we investigate the impact of the most recent large agricultural investments on the processes of urbanization and concentration of indigenous labor. We show that beyond the negative impact of

Key words

Chaco Region; Metropolitan Region of Buenos Aires; Expansion of the Agricultural Frontier; Migration;

Ethnopolitical organisation 
territorial expropriation, the breakdown of domestic economies and the labor circuits of the 20th century; indigenous populations have reoriented their migratory movements according to a regional format based on the struggle for territory, identity and the access to better living conditions.

\section{Transformações socioeconômicas, migração e organização etnopolítica rural-urbana entre a Região Chaqueña e a Região Metropolitana de Buenos Aires}

\section{Resumo}

Palavras-chave

Região de Chaqueña; Região Metropolitana de Buenos Aires; Expansão da fronteira agrícola; Migração; Organização etnopolítica
O presente trabalho tem como objetivo analisar o renovado processo de expansão da fronteira agrícola e a constante migração da população indígena para vários centros urbanos, como a Região Metropolitana de Buenos Aires, República Argentina. Pretendemos investigar o impacto que os maiores investimentos agrícolas recentes têm nos processos de urbanização e concentração do trabalho indígena. Trata-se de sustentar que, além do impacto negativo da expropriação territorial, do colapso das economias domésticas e dos circuitos de trabalho do século XX, as populações indígenas reorientaram seus movimentos migratórios em torno de novos formatos regionais baseados na luta pelo território, a identidade e o acesso a melhores condições de vida.

\section{Introducción}

El presente trabajo se encuentra dividido en tres secciones. En primer lugar, nos centraremos en desarrollar de qué manera se produjo la expansión de la frontera agropecuaria a partir de la transformación productiva y la crisis algodonera que transitó la región chaqueña durante las décadas de 1960 y 1970. En una segunda instancia, analizaremos cómo este último proceso se profundizó y consolidó desde la década de 1990 en adelante, en torno a la ejecución de políticas de corte neoliberal, de medidas extractivistas y privatizadoras. Es decir, daremos cuenta de que, en la región aquí analizada, se comenzaron a privatizar territorios fiscales y a expropiar tierras ancestrales indígenas para la incorporación de monocultivos tales como la soja. Fue a partir de esta producción que se dio inicio a una nueva dinámica de vinculaciones territoriales, modos de producción y movilidades poblacionales, como respuesta de las apropiaciones territoriales por parte de un sector de empresarios del agronegocio. En tercer lugar, analizaremos cómo se traduce el impacto que transitaron las estructuras productivas de la provincia de Chaco, en relación con los desplazamientos de población indígena hacia zonas periféricas de la Región Metropolitana de Buenos Aires (RMBA). En último lugar, cerraremos con un conjunto de reflexiones finales.

Cabe mencionar que actualmente la provincia de Chaco (mapas I y II) es el escenario de un renovado proceso de expansión de la frontera agropecuaria. Por un lado, altas inversiones de capital de carácter intensivo, la aplicación de diversas intervenciones estatales y privadas - para el desarrollo y el manejo de recursos naturales-, junto con la introducción de ordenamientos territoriales consolidan, entre otras variables, un marco en el cual se generan procesos de expulsión de fuerza de trabajo. Por el otro, y como contracara, el avance de la desforestación motiva el éxodo de la población rural y genera las condiciones para el desalojo de la población indígena y campesina (Slutzy, 2005; Iñigo Carrera, 2012). 


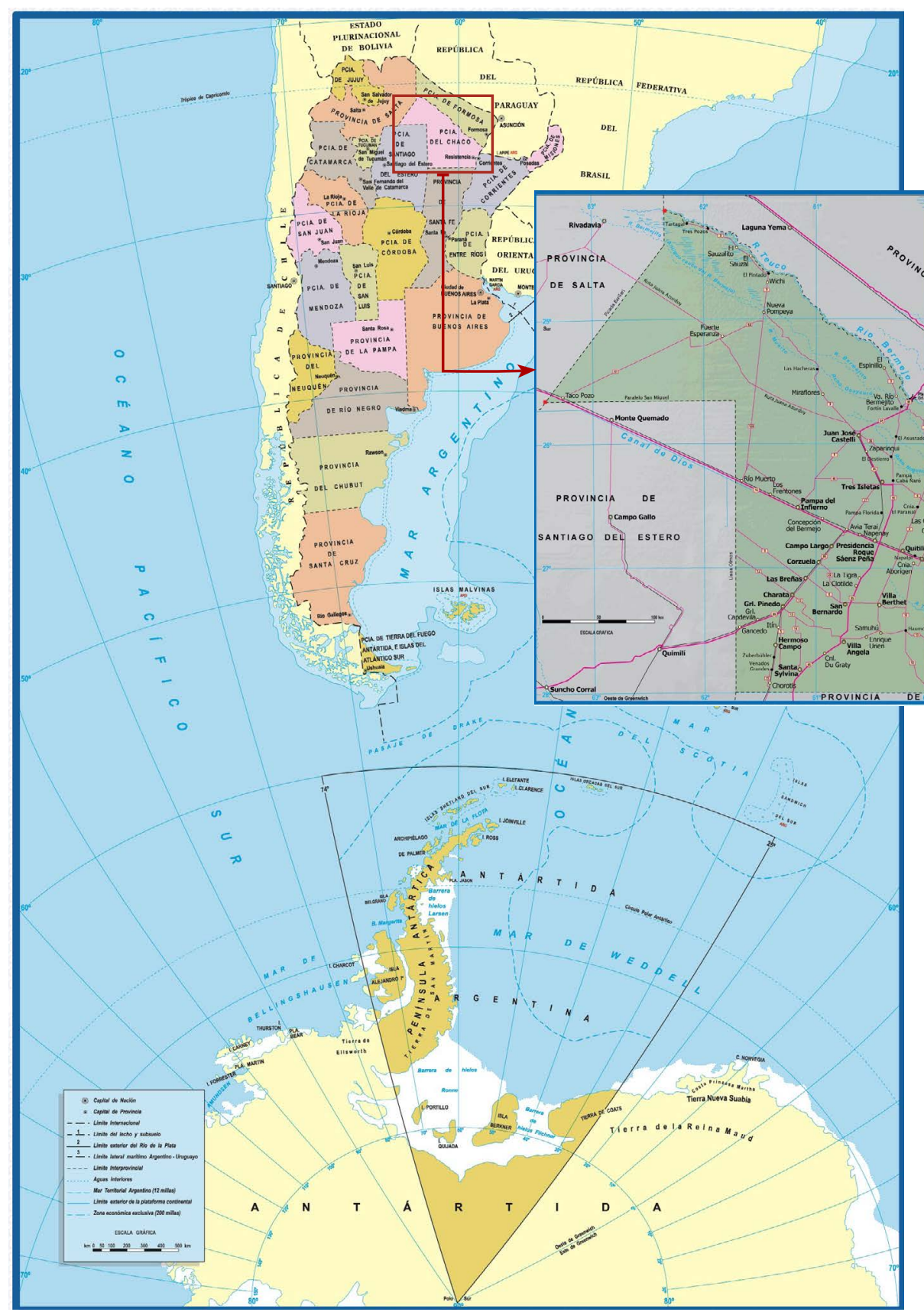

Los autores ${ }^{1}$ de este artículo pertenecemos al programa "Economía política y formaciones sociales de frontera: etnicidades y territorios en redefinición", de la Sección de Antropología Social del Instituto de Ciencias Antropológicas (ICA) de la Facultad de Filosofía y Letras (FFyL) de la Universidad de Buenos Aires (UBA). Para el desarrollo de este ensayo, por un lado, hemos recopilado fuentes primarias y secundarias, así como un conjunto de reflexiones teóricas en torno a la migración y su relación con los procesos de acumulación y concentración de la propiedad. Por otro lado, la labor etnográfica, llevada adelante en dos espacios diferentes del país desde el año 2008 (Chaco y RMBA), nos permitió, a partir del debate y su puesta en común, definir un grupo de temáticas y/o ejes analíticos que den cuenta del impacto de un proceso regional en un espacio urbano en particular. Es decir que abordar históricamente el avance de la frontera agropecuaria en la región chaqueña nos permitirá describir aquellas condiciones que incidieron en las formas de organización etnopolítica, en el acceso al
Mapa № I: Mapa de la República Argentina donde se destaca, en cuadrante rojo, la provincia de Chaco. Fuente: Instituto Geográfico Nacional.

Mapa № II: Mapa de la provincia de Chaco. Fuente: Instituto Geográfico Nacional.
1. Malena Castilla -en el marco de una beca doctoral otorgada por la Universidad de Buenos Aires- trabaja junto con las organizaciones étnicas del pueblo qom en la localidad de Pampa del Indio, provincia del Chaco, en relación con el análisis de la implementación de políticas de desarrollo. (continúa en página 103) 
2. En la década de 1920 , la provincia del Chaco comenzó a liderar el cultivo algodonero en el país y se configuró la estructura agraria que la caracterizó durante gran parte de su historia económica (Valenzuela y Scavo, 2008). (continúa en página 103)

3. Se denomina marisca a la actividad que combina la caza, pesca y recolección de frutos y miel en el monte. mercado de trabajo y en las demandas y estrategias que la población indígena llevó adelante en diferentes etapas. Pero aquello que deseamos resaltar es la multiplicidad de relaciones existentes entre ambos escenarios, para dar cuenta de la complejidad de actores y estrategias que la población indígena lleva a cabo para garantizar y efectivizar sus derechos y reclamos contemporáneos.

\section{Transformaciones socioeconómicas desde 1960 a 1980: cambios productivos y movilidad poblacional a partir de la crisis algodonera}

La incorporación histórica de los pueblos indígenas de la provincia del Chaco al trabajo se dio mediante los obrajes y, principalmente, la producción algodonera. ${ }^{2}$ Esta incorporación se realizó bajo la forma de trabajadores asalariados temporales, proletarios rurales, y como pequeños productores independientes o campesinos minifundistas. También fue común la práctica de desplazarse a los ingenios azucareros tanto dentro de la provincia del Chaco como hacia la provincia de Salta. La estacionalidad del trabajo temporal en los ingenios y en la cosecha de algodón implicaba, para estos trabajadores, la lejanía de sus hogares y/o familias, que se traducía en un ir y venir. Ese trabajo estacional se complementaba durante todo el año con la práctica de la "marisca", ${ }^{3}$ en función de conseguir mayores recursos para la subsistencia.

Entre los años 1960 y 1970 se agilizó la embestida contra esa actividad socioproductiva que les permitía a los indígenas lograr ámbitos de relativa y parcial autonomía (Gordillo, 2006) frente a las condiciones de dominación cotidianamente experimentadas. El avance de la frontera agropecuaria resultó en una creciente presión sobre los territorios indígenas. Y, por otro lado, la embestida del capital — con la consecuente concentración de propiedad de la tierra, explotación maderera y desmonte- impulsó una alta movilidad dentro de la subregión del Chaco. Ese escenario fue descripto por integrantes de diversos nucleamientos indígenas localizados en la zona sur de la RMBA durante el trabajo etnográfico.

Se trabajaba por temporada, empezaba en mayo y terminaba en noviembre. Había que trabajar mucho ahí, él iba y venía y hasta regar tenía que hacer. Yo iba solo, te iban a buscar con camiones los mayordomos [...] nos pagaban por tonelada, íbamos a la báscula y esperábamos que fuera pesado y a cambio nos daban un vale. Nos daban plata y azúcar también, pero no nos daban de comer. Yo me hacía la vianda [...] y mis compañeros eran todos 'bolitas' e indígenas, muy bien me acuerdo de eso. (Entrevista a Horacio, qom, 43 años, Rafael Calzada, provincia de Buenos Aires, agosto de 2016)

Múltiples son los relatos que describen estas experiencias cotidianas de desplazamientos al interior de la provincia, por parte de indígenas que han migrado a la RMBA desde fines de la década de 1950 en adelante. En ellos se da cuenta de que el impacto del avance del capital sobre los territorios y la población indígena actuaba de forma centrífuga sobre estos grupos familiares, pero también ponía en contacto, nucleando, a diferentes grupos y familias de diversos pueblos indígenas. En este sentido, uno de nuestros interlocutores relataba "Nos conocemos de cruzada, como somos indígenas, siempre vivimos de comunicación" (Entrevista a Teófilo, qom, 45 años, Marcos Paz, provincia de Buenos Aires), en referencia a estos movimientos de población por trabajo.

Por otra parte, la reconfiguración de la estructura productiva provincial durante las décadas de 1960 y 1980 tuvo como catalizador que la producción algodonera chaqueña - central para la economía provincial- no revistió igual importancia frente a los cultivos tradicionales de la pampa húmeda argentina (cereales y oleaginosas), que tuvieron y tienen un peso muy fuerte en el comercio agropecuario del país 
(Valenzuela y Scavo, 2008). La causa de la crisis de la producción algodonera nacional que tuvo lugar hacia fines de la década de 1960 puede encontrarse en numerosas variables: la desarticulación en el territorio de cadenas de valor agregado, el proceso de tecnificación y la diversificación hacia otros cultivos, principalmente hacia la soja que avanzo sobre hectáreas antes destinadas al cultivo del algodón ${ }^{4}$ (García, 2007).

Las políticas neoliberales aplicadas por la dictadura militar durante la década del setenta $-\mathrm{y}$ continuadas durante los años noventa- profundizaron estas transformaciones. Entre fines de los años setenta y comienzos de la década del ochenta, empresas textiles radicadas en el Chaco se dirigieron a otras provincias más favorecidas por la política de promoción industrial (San Luis, La Rioja, Catamarca, San Juan y Tucumán), donde los eslabones industriales (textiles y confecciones) se asentaron para aprovechar estímulos fiscales, lo que hizo que el complejo algodonero quedase definitivamente desdoblado (García, 2007). Respecto del tamaño de las explotaciones algodoneras en el Chaco, el número de los grandes productores fue en aumento a partir de los años sesenta del siglo XX (Pertile, 2004). Estos procesos de cambio se manifestaron en la imposibilidad de los minifundistas de sostener una producción rentable y en la pérdida de numerosas fuentes de trabajo, directas e indirectas. La crisis de los años sesenta, ya mencionada, la mecanización del agro en general a partir de los años setenta y el aumento de la incorporación de la cosechadora mecánica en el cultivo de algodón desde fines de la década del ochenta provocaron que aquellos indígenas que habían sido incorporados como trabajadores en su producción acabaran por constituirse en mano de obra sobrante, imposibilitados de mantenerse a sí mismos y a sus familias. Durante este período, a la par de las migraciones hacia ciudades distintas del propio Chaco, en forma paralela, se acrecentaron las migraciones hacia ámbitos metropolitanos por fuera de los límites provinciales, tales como las provincias de Santa Fe y Buenos Aires.

\section{Profundización y consolidación de la frontera agropecuaria en la década 1990: proceso de sojización a partir de privatizacio- nes y usurpaciones territoriales}

En este segundo apartado nos centraremos en analizar de qué manera se efectuó el proceso de expansión de la frontera agrícolo-ganadera durante la década de 1990 en la provincia de Chaco. Para ello, nos centraremos en explicitar aquellas políticas que fueron ejecutadas a partir de las privatizaciones de los campos, y así comprender el alto nivel de movilidad de la población rural hacia zonas urbanas.

Tal como describimos en el acápite anterior, desde la década de 1970 se inició un proceso de corrimiento y expansión de la frontera agraria de la soja hacia zonas de bosques y montes del país (Giarracca y Teubal, 2010). La incorporación de este producto a gran escala en los cultivos tuvo lugar a partir de la fuerte demanda internacional respecto de otras manufacturas agrícolas y/o ganaderas de Chaco.

Durante la década del noventa, este modelo de transformación económica se profundizó a partir de la implementación de diversas políticas globalizadoras de corte neoliberal que acompañaban la expansión de las fronteras del libre mercado sobre los Estados soberanos. Entre ellas, se destacaban el fomento de la inversión extranjera, la apertura comercial, la privatización de empresas y servicios del orden público y territorios nacionales, además de la desregulación estatal.

Estas medidas, implementadas en todo el territorio nacional, incidieron de diversas maneras en la provincia de Chaco: entre los años 1994 y 2007, la provincia perdió el $80 \%$ de las tierras fiscales a manos de sociedades anónimas y empresarios dedicados a la producción agrícola y ganadera. Según los datos del Instituto de Colonización, ${ }^{5}$ el
4. De acuerdo con Rosati (2008), al analizar las series de tiempo de superficie y producción algodonera provinciales se pueden identificar cuatro momentos de crisis: el primero, entre 1965/66 y 1971/72; el segundo, entre 1977/78 y 1981/82; luego, entre 1989/90 y 1992/93; y el último, entre 1995/96 hasta 2003/o4 (este último periodo lo analizaremos en los próximos apartados).
5. Organismo de orden provincial, encargado de regular y administrar las tierras fiscales de Chaco. 
6. En este sentido, la primera compra de tierras que realizó dicha empresa fue a la compañía Bunge \& Born, que poseía territorios que habían sido parte de la apropiación que realizó Victorica tras la campaña del año 1884 en la provincia de Chaco (Zorzoli, 2016).

7. De hecho, a mediados del año 2017, UNITEC absorbió las empresas América Lodging SA y El Tacurú SA, las cuales poseían grandes extensiones de tierras (Boletín Oficial, 2017).
Gráfico №1: Elaborado a partir de los datos de los censos 2001 y 2010 (INDEC, 2001, 2012). estado chaqueño pasó de tener 3 millones y medio de hectáreas en el año 1994, a 650 mil en diciembre del 2007 (Muñoz y Gallo, 2012).

La privatización del territorio provincial marcó el ingreso de grandes empresarios del agronegocio a la región. Dicho proceso implicó la puesta en marcha de transformaciones en los cultivos locales destinadas a garantizar la explotación de las producciones exportables. Durante la década del noventa se generó la expansión de la soja genéticamente modificada, lo cual no solo significó la reconversión productiva, sino que forzó la expansión de la frontera agrícola. Es decir, los desmontes indiscriminados en la zona para la siembra de este monocultivo produjeron la migración de la población qom, wichi y moqoit, entre otros, en el marco de un proceso de apropiación y exclusión territorial (Stoler, 1987). Además, es importante remarcar que prácticas como la marisca mermaron en gran parte de las comunidades debido a la usurpación de los montes nativos y a la pérdida de biodiversidad derivada de las nuevas producciones, los desmontes y las fumigaciones.

En la localidad chaqueña de Pampa del Indio, el proceso de concentración de la propiedad puede ser ejemplificado a partir del caso de la estancia "La Leonor". Esta forma parte de la empresa UNITEC AGRO, la cual adquirió más de 70 mil hectáreas durante diferentes negociaciones entre empresarios. ${ }^{6}$ Otra de las compras que realizó UNITEC fue durante la década de 1990, con la expansión de la frontera agroganadera. Esta empresa comenzó a trabajar en la producción de algodón a partir de la utilización de semillas transgénicas y sistemas mecanizados de riego, entre otras tecnologías. Aunque en la provincia de Chaco UNITEC posee formalmente más de 90 mil hectáreas, ese número - según las entrevistas y observaciones etnográficas realizadas - se incrementa día a día, dado que la línea de explotación no ha dejado de avanzar sobre los territorios antiguamente pertenecientes a campesinos e indígenas, que, desde la década del noventa hasta el presente, comenzaron a vender sus tierras a bajo costo ${ }^{7}$ (Luna, 2016).

Hacemos mención a este caso para ejemplificar como se da la venta y apropiación de tierras en la región, lo cual no solo trae aparejado el cambio productivo de la zona, sino también la expulsión de comunidades indígenas hacia diversos ámbitos urbanos. El gráfico N. ${ }^{\circ} 1$ enseña, además, las caídas porcentuales de población rural en la provincia de Chaco desde 1914 hasta 2010:

\section{Caída demográfica en ámbitos rurales}

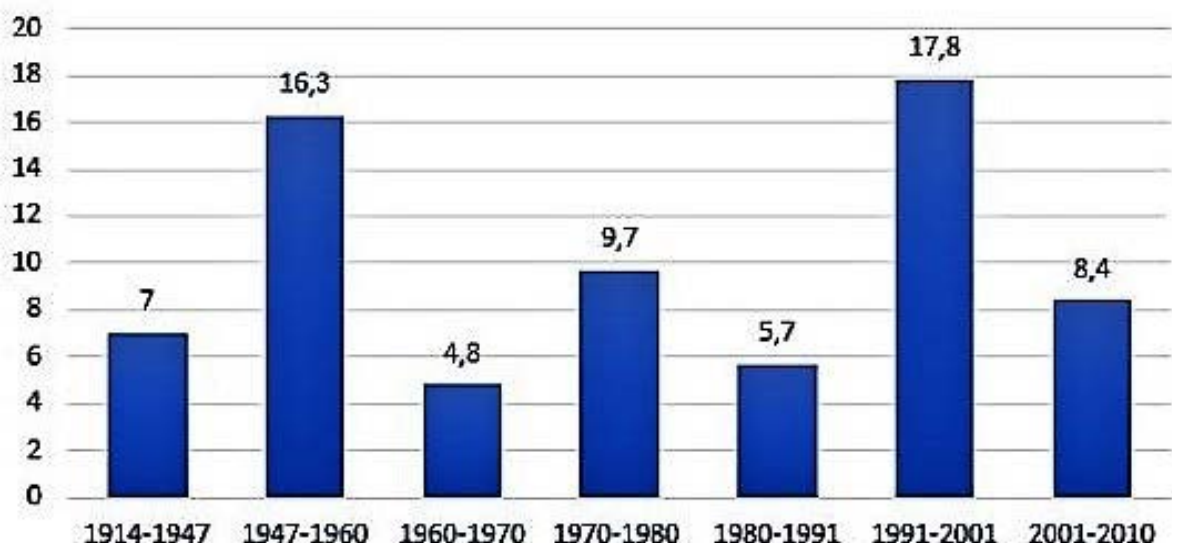

De la lectura de estos datos surge que la población rural se redujo, fundamentalmente, entre los años 1991 y 2001; mientras que la población indígena urbana se incrementó (en el mismo período) (INDEC, 2001). Estos datos indican efectivamente que se ha 
producido en los últimos años un continuo proceso de urbanización provincial, dadas las migraciones internas a los grandes centros urbanos de Chaco como son Resistencia, en primer lugar, y Presidencia Roque Sáenz Peña, en segundo lugar (Ebel, 2013).

Resulta concluyente que desde finales de la década del ochenta hasta la crisis socioeconómica del año 2001, las políticas económicas neoliberales se intensificaron en la provincia chaqueña producto de la llegada de multinacionales del agronegocio, que obtenían hectáreas para el cultivo a partir de la negociación con el empresariado local y el estado provincial (Castilla, 2017). De esta manera, mientras en el año 1995 había 4 millones de hectáreas que correspondían a los pueblos indígenas y pequeños productores, en 2007 solo quedaban 660 mil hectáreas (Attias y Lombardo, 2014).

Este contexto socioeconómico, que incrementó la desigualdad y restringió el acceso a la propiedad, estuvo nutrido por diversos programas paternalistas y de asimilación legitimados por agencias de cooperación internacional en el marco del llamado "multiculturalismo" (Hale, 2002). De este modo se pusieron en práctica en Argentina programas de asistencia y de desarrollo a cargo de diferentes agencias gubernamentales sostenidas por diversas fuentes de financiamiento internacional. Es de este modo, que las organizaciones no gubernamentales (ONG) se presentarían durante la década de 1990 en la región chaqueña con proyectos de desarrollo entre cuyos objetivos figuraba generar procesos de inclusión de la población explotada, tanto indígena como no indígena. A pesar de que las políticas neoliberales se sustentaban en un aparato conceptual que propugnaba las libertades individuales (Harvey, 2013), las condiciones que generaba este modelo impulsaron, paradójicamente, la creación de espacios de resistencia en los cuales participaban los sujetos explotados en pos de producir un cambio social. Las condiciones de precariedad laboral, la expropiación de los territorios, el incremento de la violencia y la discriminación intensificaron las reivindicaciones y fomentaron un proceso de organización tanto en Chaco como en la RMBA (aspecto que veremos en la próxima sección).

En este sentido, gran parte de las organizaciones etnopolíticas han profundizado su trabajo durante las décadas de 1990-2000, en relación con los escenarios socioeconómicos y políticos que se produjeron durante esta época. Cabe mencionar que en décadas anteriores se habían conformado diversas agrupaciones étnicas (Domínguez, 2005; Harvey, 2007; Iñigo Carrera, 2012; Weiss, Engelman y Valverde, 2013) que durante este período consolidaron su estructura política.

\section{Formatos de desplazamiento y migración regional de población indígena en la actualidad}

En este tercer apartado nos proponemos caracterizar aquellas modalidades que hoy en día definen a los desplazamientos y migraciones de la población qom, en una escala regional que incluye tanto localidades de la provincia de Chaco como de la RMBA. En términos históricos, el proceso de expulsión, acompañado por la expropiación territorial y la violencia directa e indirecta sobre las familias indígenas, provocó, junto con el quiebre de las economías domésticas, el abandono del lugar de origen (Slutzy, 2005; Iñigo Carrera, 2012). La búsqueda de mejores condiciones de vida y la inserción de la población en circuitos de trabajo - tanto formal como informal- fueron motivos y anhelos a partir de los cuales vastos contingentes de población migraron hacia zonas urbanas. A modo de ilustración, y de acuerdo con datos demográficos, ${ }^{8}$ el pueblo qom se ubica en su mayoría a lo largo del corredor geográfico y comercial que une a las ciudades de Resistencia, Rosario, Buenos Aires y La Plata. Dicho eje, al tiempo que enseña la concentración de población indígena, expresa el papel que posee la urbanización en la absorción de capital (Harvey, 2013). Para comprender la
8. De acuerdo con los datos provistos por la Encuesta Complementaria de Pueblos Indígenas (INDEC, 2005), aproximadamente un $68 \%$ de la población Toba (qom) se concentra en diversas áreas urbanas del país (Weiss et al., 2013). 
9. En los años setenta, en la ciudad de Resistencia, unas 220 familias qom accedieron a un plan de viviendas del municipio mediante el cual se formalizó el "Barrio Toba". (continúa en página 104)

10. El “Nuevo Barrio Toba” está conformado por población qom, moqoit y población criolla, y su creación ha sido resultado de una relocalización de diversas familias indígenas llevada a cabo por la Municipalidad y el Servicio Público de la Vivienda (Vázquez, 200o).

11. Dicha reestructuración, sustentada en la hegemonización del paradigma neoliberal e instaurada con el golpe cívico-militar de 1976, (continúa en página 104)

12. Las "villas miseria" o "de emergencia", ganaron terreno por sobre otras modalidades de acceso a la tierra y vivienda para los sectores populares (Torres, 1992, 1993). (continúa en página 104)

13. Respecto de la duplicación de los asalariados precarizados definidos como aquellos a quienes no se les realizan los descuentos jubilatorios-, (continúa en página

104)

14. La tasa de desocupación de dicho aglomerado, de acuerdo con Maceira (2012), creció entre el 6,3\% y el $16,4 \%$ de la Población Económicamente Activa entre 1991 y el 2003, (continúa en página 104) contracara de ese proceso de urbanización, David Harvey sostiene que la creación de la nueva geografía urbana supone inevitablemente desplazamientos, así como también la desposesión (Harvey, 2013). En el caso de la población qom, y la migración al interior de ciudades de la provincia de Chaco, ${ }^{9}$ podemos mencionar que esta comenzó a fines de la década de 1950 en localidades tales como Sáenz Peña, Castelli, Resistencia y Las Palmas (Wright, 1999). Para la ciudad de Rosario - capital de la provincia de Santa Fe- dicho fenómeno tuvo lugar durante la década de 1960, a partir de la conformación de diversos "Barrios Tobas" como Villa Banana, Cerrito, Empalme Graneros, Los Pumitas y el llamado "Nuevo Barrio Toba" ${ }^{10}$ en la periferia de la ciudad (Bigot, Rodríguez y Vázquez, 1991, 1992 y 1995; Garbulsky 1994; Viglianchino, 1997a, 1997b; Cardini, 2006). En el caso de Buenos Aires y la ciudad de La Plata, durante la década de 1960 se produjo el proceso migratorio y de llegada de varias familias qom hacia la llamada "Villa IAPI", ubicada en el partido de Quilmes, al sur de la Ciudad Autónoma de Buenos Aires (CABA) quienes, posteriormente y como corolario de un proceso organizativo, conformaron el "Barrio Toba" de Las Malvinas (Tamagno, 2001, 2014; Maidana, 2011). Liliana Tamagno hace referencia a un dato que permite visualizar el proceso de desposesión y expulsión por avance del capital, migración y urbanización, al mencionar que en el Censo Nacional de Villas del año 1967, un 80\% de la población de la "IAPI" era proveniente de la provincia de Chaco (Tamagno, 2014).

En relación con estos datos, quisiéramos realizar unas breves reflexiones. Nuestro trabajo etnográfico con diversos integrantes de nucleamientos qom localizados en la RMBA nos permite considerar que el grueso de los migrantes arribó a dicho ámbito entre las décadas de 1960 y 1980. Este desplazamiento interno se relacionó con una importante expansión de la periferia urbana, cimentada en tres factores: el acceso a la tierra urbana y a la vivienda, la radicación de la industria y el desarrollo de la red de transporte (Maceira, 2012). Como ya describimos, durante la década del sesenta, y especialmente a partir de los años setenta, se produjo una reorientación en las políticas del Estado, lo cual produjo una reestructuración de la economía, ${ }^{11}$ con el consecuente debilitamiento de la expansión periférica en un contexto de aumento de la desindustrialización y de deterioro de las condiciones habitacionales. ${ }^{12}$

En este sentido, es importante considerar que la reestructuración económica mencionada, si bien tuvo un impacto diferencial en la RMBA y en la provincia del Chaco, constituyó un mismo proceso de concentración del capital, que alcanzó su momento más álgido durante los años noventa. El paradigma neoliberal desde el que se puso en marcha la reforma desreguladora del Estado, sustentado en una visión empresarial de la gestión pública y en la transferencia de servicios públicos al sector privado o "tercer sector", intensificó aún más la desigualdad social y la precarización de las condiciones de vida de grandes conjuntos sociales. Respecto de ello, entre los años 1974 y 2003, estudios que han procesado y analizado los datos de la Encuesta Permanente de Hogares (EPH) señalan un incremento de la precarización laboral para el Aglomerado GBA (Ciudad de Buenos Aires y Conurbano) que duplicó la proporción de asalariados precarios, ${ }^{13}$ así como un marcado incremento de la desocupación. ${ }^{14}$ El aumento del trabajo precario afectó de modo más intenso a los segmentos de trabajadores ya desfavorecidos, a aquellos insertos en puestos de menor productividad y a los asalariados de menores ingresos (Maceira, 2012; Calvi y Benza, s/f). En la RMBA, todo esto conllevó a una reconfiguración de la dinámica de estructuración urbana caracterizada por una creciente desigualdad en el acceso a la ciudad (Harvey, 2013), y particularmente a la tierra y la vivienda, lo que se complementó con una precarización laboral también en aumento y una profunda crisis de empleo, reflejada en las crecientes dificultades que se encontraron los migrantes qom al momento de insertarse en empleos formales. 
Como contracara de este proceso, si bien en la provincia del Chaco se intensificó la expansión de la frontera agropecuaria y se concentró marcadamente la propiedad, en el transcurso de la década de 1990 - como analizamos en el segundo acápitetuvo lugar una transformación en la modalidad migratoria y de desplazamiento de la población indígena. En este sentido, parte de este cambio puede responder al dato de que la RMBA, por lo antedicho, dejó de ser concebida como un lugar de fuerte recepción para la migración interna en general y la indígena en particular. Antes bien, estos desplazamientos adquirieron nuevos formatos y se orientaron hacia las periferias de centros urbanos del espacio provincial como Resistencia u otras localidades próximas.

Lo que nos interesa resaltar es que, si bien los indígenas ya no solo migraban definitivamente a la RMBA como en décadas anteriores, a partir de la década de 1980 y durante los años 1990, los desplazamientos entre las provincias del Chaco y Buenos Aires adquirieron dinámicas que fortalecieron entre los integrantes los lazos de nucleamientos, comunidades y organizaciones indígenas. Este fortalecimiento, sustentado en una práctica etnopolítica, logró protagonismo a la luz de transformaciones en el marco jurídico, y del proceso de descentralización y desregulación estatal. Asimismo, consideramos que en el refuerzo de los lazos etnopolíticos entre ambas provincias y en las transformaciones de las formas de desplazamiento indígena, también ha jugado un rol cardinal la implementación de políticas públicas. Es decir, el proceso de descentralización y desregularización estatal experimentado durante los años noventa - con la reforma constitucional de 1994- y comienzos de la década del 2000, en un contexto de políticas de ajuste estructural, se plasmó en la implementación de un conjunto de políticas públicas frente al aumento de los niveles de desempleo y pobreza. Se aplicaron así programas de ayuda vinculados al desempleo - tal como mencionamos en el apartado anterior-, financiados por organismos de crédito internacionales como el Banco Interamericano de Desarrollo (BID) y el Banco Mundial (BM). En paralelo a estos programas, también se desarrollaron otros, dirigidos a atender el déficit alimentario. Durante aquellos años, la política estatal frente a la desocupación estuvo destinada a la puesta en marcha de programas de ocupación transitoria o "planes", y el gasto social se focalizó en aquellos grupos sociales y aquellas regiones calificadas como "pobres" (Manzano, 2013). A la vez, esta configuración de estatalidad a partir de los mencionados programas sociales se deslindaba de responsabilidades, que fueron traspasadas, no solo a la gestión privada o de ONG, sino también hacia organizaciones sociales y comunitarias. La interrelación de diversas agencias estatales y la acción colectiva en la implementación de estos programas conllevó a su vez altos niveles de politización de los actores sociales involucrados. Por otra parte, destacar aquellas modalidades migratorias que dinamizaron y caracterizaron a los desplazamientos indígenas de la década de 1990 en adelante demanda también retomar el marco jurídico y de reconocimiento de derechos específicos. Este modelo, que Hale denominó "multiculturalismo neoliberal" (2002), fue definido, a grandes rasgos, como un fenómeno en el cual el liberalismo era reelaborado para reconstruir una estrategia de "inclusión" de la diferencia que fuera compatible con el capitalismo globalizado (Díaz Polanco, 2006). Bajo esa premisa, las demandas culturales indígenas son aceptadas como parte de los procesos de democratización y gobernanza, a la vez que se limita la redistribución de los recursos y del poder (Hale, 2002; Stavenhagen, 2002; Assies, 2009; De la Maza Cabrera, 2012). Sin embargo, y paradójicamente, dicha dinámica propició espacios de participación política en la propia estructura estatal. Estos espacios, desde los cuales la población indígena fue adquiriendo mayor protagonismo en la implementación de políticas, permiten comprender los nuevos formatos que adopta la relación entre Estado-sociedad, así como aquellas instancias de reorganización étnica y etnopolítica. 
Quienes arribaron al tejido periurbano de la RMBA en los años sesenta, setenta y, en menor medida, en los ochenta, relatan instancias en que parientes del interior fueron recibidos en sus hogares de forma asidua.

Yo, gracias a ella [su tía materna], conocí Buenos Aires, me vine por ella. Me vine para acá con Roberto y con su señora, Rita [Hermano y cuñada de la mujer mencionada], un agosto, hacía un frío... Pleno frío. No sabía el frío que hacía. Me vine con toda ropita de verano. Bajamos en Retiro. Veníamos en tren Roberto, la señora, que traía a su hija, tenía más o menos 10 días, era muy bebita. Llegamos en el 148 acá, que yo no conocía nada. Y vivimos en la casa de mi tía en los primeros tiempos. Bajamos en la estación de Solano; mi tía ya sabía que veníamos, preparó asado. Después de ahí conocimos Buenos Aires. Había muchas chicas en la casa de mi tía, todas primas, ¿vio mi prima Irene? Era muy jovencita y trabajaba cama adentro y venía los sábados con varias primas. Mi tía no tenía esa casa que tienen ahora, tenía una casilla, y ahí dormíamos todos. Y después mi prima trabajaba y me dice '¿Mari querés trabajar en casa de familia, cama adentro?' en Palermo. (Entrevista a Raquel, qom, Rafael Calzada, agosto 2016)

El presente testimonio no solo expresa un proceso migratorio individual, sino que da cuenta de las condiciones y la importancia que las relaciones de parentesco poseen tanto en su papel receptor como en la búsqueda de trabajo. No obstante ello, en la actualidad, podemos decir que una de las características que definen a las migraciones es la posibilidad de "ir y volver" desde la ciudad, como desde la localidad de origen. Para mencionar un caso, Weiss describe cómo la comunidad "Nogoyin Ni Nala" de Rafael Calzada (Almirante Brown), en el año 1998, comenzó a definir la necesidad de crear una "delegación" de la reconocida Asociación Indígena de la República Argentina (AIRA) (Weiss, 2015).

Cabe destacar que, durante los años 1990, la AIRA fue una de las organizaciones indígenas que, junto con el Centro Kolla, tuvieron un rol pivote entre la población indígena en el área metropolitana; e incluso contribuyó a apuntalar experiencias organizativas y afirmativas en otras provincias del país. Encolumnados en AIRA, referentes de diversos nucleamientos indígenas de la RMBA habían participado de las negociaciones por la sanción de la Ley Nacional 23.302 de 1985, así como también ejercieron su "capacidad de lobby", junto con otras organizaciones indígenas del país, para lo que posteriormente sería la reforma constitucional de 1994. Esta organización llegó a ser concebida como un "Ministerio de los indígenas" para muchos de aquellos que recién llegaban a la CABA. Por ejemplo, Sergio, integrante de una comunidad qom en Marcos Paz, relata que cuando llegó a Buenos Aires proveniente de la provincia del Chaco, en el año 1986, se hospedó en AIRA, ya que no contaba con otro lugar donde vivir.

Y el AIRA era como un lugar... un lugar para hacer trámites, ponele. Rogelio Guanuco [presidente de la Asociación] quería que las comunidades participen, hagan reuniones. Había camas, colchones, venían de todos lados, de Salta, Formosa, del sur también, los hermanos mapuche, de Jujuy, de Salta los hermanos wichí. La mayoría siempre venían de Chaco y de Salta. Por ahí mucha gente de Tartagal. Y más que nada venían por el tema de la tierra. (Entrevista a Sergio, qom, Marcos Paz, marzo 2016)

Por su parte, Matías, integrante de la comunidad qom de Marcos Paz, recuerda al AIRA como un punto neurálgico para el movimiento indígena:

A lo mejor viven lejos, llegan de todos lados a AIRA, paraguayos, bolivianos, de Salta, llegan los abogados, todos altos cargos. Se hacía reunión los sábados hasta las 12 de la noche, para comer. Entre AIRA y Centro Kolla, nos íbamos a visitar, siempre. (Entrevista a Matías, qom, Marcos Paz, junio 2016) 
Los testimonios citados, al tiempo que dan cuenta del tipo de actividad que diversos dirigentes llevaban a cabo en la CABA, expresan el nivel de concentración que esta posee en torno a organismos e instituciones que tienen por objetivo garantizar la aplicación y la consulta de los derechos indígenas. La realización de trámites, y el carácter administrativo que adopta la relación entre el Estado y las poblaciones indígenas, convirtieron al INAI, al AIRA y demás organizaciones en ámbitos donde migrantes indígenas de distintos pueblos cruzan sus caminos y sus experiencias. Podemos decir que esos espacios, al igual que el contacto permanente entre los distintos nucleamientos entre sí, enriquecieron los procesos de toma de conciencia sobre derechos indígenas y posibilitaron la continuidad de encuentros, reuniones y la realización de festividades entre los "hermanos".

Por otra parte, las familias que migraron primariamente hoy en día se han constituido como una suerte de "colchón receptivo" que dinamiza este tipo de migraciones de "ida y vuelta". Hay quienes desde el interior del país arriban a la CABA y a la RMBA con el objetivo de concurrir a organismos institucionales, visitar familiares, trabajar temporalmente en el espacio urbano, asistir a festividades y conmemoraciones en sedes de la Iglesia evangélica o, por ejemplo, participar de festividades importantes (como el 19 de Abril, "Día del Aborigen Americano"). De la misma forma, también se producen desplazamientos recurrentes de los qom desde la RMBA hacia los lugares donde se asientan sus familiares, tanto para participar de las mencionadas experiencias en sus territorios de procedencia o en aquellos procesos políticos que estén teniendo lugar. De hecho, y a modo de ejemplificación, el dirigente político visible de un nucleamiento qom/moqoit se constituye como tal en una fuerte y continua movilidad entre Rafael Calzada y distintas comunidades de la provincia del Chaco donde se encuentran asentados sus numerosos parientes moqoit. Asimismo, los parientes situados en la región metropolitana realizan gestiones de diversos proyectos - de viviendas o productivos- en organismos estatales situados en Buenos Aires a nombre de sus parientes localizados en la provincia del Chaco. Otras veces, los propios parientes, muchos de ellos dirigentes, viajan y hacen diversos trámites, mientras se hospedan en la ciudad. A su vez, del Chaco llegan "hermanos" que traen consigo artesanías para comercializarlas en distintos puestos en ferias y templos evangélicos. Como podemos ver, este formato de desplazamiento basado en múltiples "visitas" entre parientes y amigos que viven en una u otra provincia, confiere un gran dinamismo al proceso etnopolítico, y al mismo tiempo expresan el carácter regional de los movimientos indígenas del presente. A esto se debe sumar la implementación de las nuevas tecnologías de comunicación y difusión, en el marco de estrategias políticas y formas de disputar recursos estatales.

Por otro lado, cabe mencionar que ello encuentra sustento gracias al avance que la reforma de la Constitución nacional del año 1994 tuvo en torno a los reclamos territoriales y a la política de reconocimiento de personerías jurídicas indígenas de las últimas dos décadas. Este hecho no solo permite, más allá de la complejidad y conflictividad que genera, una mayor resistencia en los espacios de origen, sino que requiere de una dinámica, y un tipo de migración constante, para realizar trámites y presentar documentaciones en la dependencias institucionales urbanas. Finalmente, otro factor que imposibilita el traslado definitivo de población indígena hacia la periferia de la RMBA lo encontramos en el incremento del valor de la propiedad y la escasez de tierras disponibles.

\section{Consideraciones finales}

La migración rural-urbana de población qom en los últimos años ha tomado un carácter regional a partir del marco de derechos y del uso estratégico que estos permiten a 
la hora de garantizar e implementar estrategias para mejorar las condiciones de lucha y negociación por los territorios indígenas.

En las presentes páginas hemos señalado que, si bien la expansión de la frontera agropecuaria y ganadera ha afectado a la provincia de Chaco desde la década de 1990, dicha expansión debería de haber impactado en la expulsión de la población indígena de sus territorios. Sin embargo, tal como describimos, este proceso no se dio de esta manera en los años de 1990 como sí ocurrió en décadas anteriores. Es decir, a diferencia de la mitad del siglo XX, cuando las migraciones tenían un carácter terminal, en la actualidad vemos múltiples modalidades por las cuales los dirigentes y miembros de comunidades indígenas hacen un uso de sus desplazamientos entre los lugares de origen y las zonas urbanas - ya sea la capital provincial, nacional o la CABA - con fines de realizar diversos tipos de labor. Esto último, motivado por la aplicación de programas y políticas públicas marca una tendencia, por un lado, de una organización etnopolítica arraigada. Por otro lado, se puede reconocer en estas migraciones actuales que "la ida y vuelta" de población indígena se realiza de manera constante. Cabe señalar también la importancia que tienen en este escenario las nuevas tecnologías, así como la propia dinámica de conexión nacional que estas han posibilitado en los últimos años. Podemos decir que este tipo de migración — regional y de "ida y vuelta" - es parte de un proceso de dinamización de relaciones de un campo etnopolítico que, si bien actúa localmente, lo hace a través de horizontes más amplios.

Tal como analizamos en este artículo, las lógicas de acumulación contemporáneas difieren de aquellos modelos extractivos rurales en beneficio de la expropiación de tierras, de la explotación de mano de obra indígena y del agotamiento de la producción de monocultivos tales como el algodón o la caña de azúcar. Actualmente, la cotidianeidad de vastos contingentes indígenas, ya sean rurales o urbanos, se ancla en reclamos que surgen de procesos consecuentes de la misma lógica de acumulación del capital. Es decir que la propia dinámica de expansión de la frontera agropecuaria, en términos de concentración de propiedad y en el marco de derechos, permite que se genere un escenario que da sentido a la acción colectiva, y por extensión, a las maneras de hacer política de la población étnica.

\section{Financiamiento:}

Este documento es resultado del financiamiento otorgado por el Estado Nacional, por lo tanto queda sujeto al cumplimiento de la Ley № 26.899. Asimismo, se realizó en el marco de las investigaciones promovidas por el Proyecto de la Programación Científica UBACyT 2014-2017 (cod. 20020170100294BA), Argentina: "Movilizaciones indígenas y de pequeños productores criollos: Conflictividad territorial, transformaciones regionales, trayectorias sociohistóricas y reconfiguraciones étnico identitarias"; el proyecto UBANEX gna. Convocatoria, Argentina: "Indígenas en la ciudad. Articulación y fortalecimiento de espacios etnopolíticos en el Área Metropolitana de Buenos Aires"; el PICT 2015-3650, Argentina: "Conflictividad territorial y etnicidad Mapuche en Norpatagonia Argentina: transformaciones socioeconómicas, políticas regionales"; y el PDTs CIN 262, Argentina: "Del territorio a la ciudad: características socioculturales, trayectorias de la migración y políticas públicas en relación a los pueblos indígenas en ámbitos urbanos" de la Universidad Nacional de Luján.

\section{Agradecimientos:}

En primer lugar deseamos agradecer la buena predisposición de todas y todos aquellos que nos recibieron durante nuestro trabajo etnográfico tanto en la provincia de Chaco 
como en la Región Metropolitana de Buenos Aires. Ello ha posibilitado una labor colaborativa, la cual enmarca el conjunto de las reflexiones presentes. En segundo lugar al equipo y a los directores del Programa de "Etnicidades y territorios en redefinición", quienes enriquecieron este manuscrito con sus aportes. Finalmente, no queremos dejar de mencionar a la Dra. Liliana Landaburu, que compartió con nosotros diferentes instancias de trabajo y de compañerismo, así como de docencia y amistad

\section{Sobre los autores}

Malena Inés Castilla es licenciada en Antropología Social por la Universidad de Buenos Aires (2013). Desarrolla desde el año 2011 su investigación junto a las organizaciones indígenas de la localidad de Pampa del Indio Chaco. Es becaria doctoral de la Universidad de Buenos Aires.

María Laura Weiss es profesora en Ciencias Antropológicas (2012). Desde el año 2013 realiza su investigación sobre el proceso etnopolítico del pueblo Qom, la reconfiguración de su dirigencia y modalidades de participación estatal en la Región Metropolitana de Buenos Aires. Es becaria doctoral del Consejo Nacional de Investigaciones Científicas y Técnicas (CONICET).

Juan Manuel Engelman es doctor en Antropología Social por la Universidad de Buenos Aires (2017), y desde hace una década investiga acerca de procesos de participación etnopolítica en gobiernos locales de la Región Metropolitana de Buenos Aires. Es becario posdoctoral del CONICET.

\section{Notas}

1 Malena Castilla -en el marco de una beca doctoral otorgada por la Universidad de Buenos Aires- trabaja junto con las organizaciones étnicas del pueblo qom en la localidad de Pampa del Indio, provincia del Chaco, en relación con el análisis de la implementación de políticas de desarrollo. María Laura Weiss realiza su investigación doctoral -con una beca del CONICET - sobre el proceso etnopolítico del pueblo qom, la reconfiguración de su dirigencia y formas de participación política en la RMBA; y Juan Manuel Engelman -becario posdoctoral del CONICET— analiza desde el año 2008 procesos de organización etnopolítica en gobiernos locales de la RMBA. (En página 93.)

2 En la década de 1920, la provincia del Chaco comenzó a liderar el cultivo algodonero en el país y se configuró la estructura agraria que la caracterizó durante gran parte de su historia económica (Valenzuela y Scavo, 2008). El avance de esta producción acompañado por un aumento de migraciones de braceros en tiempos de cosecha, la utilización de mano de obra de la industria forestal en retirada, el surgimiento de nuevos centros urbanos y el crecimiento del sector de servicios. La colonización agrícola impulsada por el Estado nacional llevó a que cientos de agricultores se ubicaran en las colonias oficiales y privadas. Las áreas algodoneras por excelencia fueron los sectores sudoeste, centro y oriental - que abarca los departamentos de Comandante Fernández, Chacabuco, 12 de octubre, Fray Justo Santa María de Oro, General Belgrano, Maipú, Fontana Coronel Du Graty, 9 de Julio y $\mathrm{O}$ Higgins-. Este fue el cultivo por excelencia de minifundistas y pequeños y medianos productores (Carlino, 2001; García, 2007; Valenzuela y Scavo, 2008). En ese primer momento, su producción se dirigió hacia el mercado externo, mientras que fábricas de aceite y desmotadoras se instalaron en esta zona. Desde mediados de los 
años 1930, como resultado de la crisis mundial, se reorientó dicha producción hacia el mercado nacional. El destino de la producción algodonera durante el modelo sustitutivo estuvo marcado por el desarrollo y expansión de la industria textil nacional, la cual, para 1954, era la segunda en importancia en el conjunto de las industrias manufactureras, después de las alimenticias (Carlino, 2001). (En página 94.)

9 En los años setenta, en la ciudad de Resistencia, unas 220 familias qom accedieron a un plan de viviendas del municipio mediante el cual se formalizó el "Barrio Toba". En ese mismo período, este hecho también se registra en las periferias de algunas ciudades de la provincia de Formosa, donde numerosas familias qom accedieron a planes de viviendas municipales (Ibáñez Caselli, 2002 citado en Maidana, 2011). (En página 98.)

11 Dicha reestructuración, sustentada en la hegemonización del paradigma neoliberal e instaurada con el golpe cívico-militar de 1976, tuvo por objetivo la apertura económicofinanciera caracterizada por la centralidad de la exportación del sector agropecuario y un grupo reducido de actividades industriales, lo cual impactó negativamente sobre las economías "regionales". Otros de sus efectos buscados fueron la contracción del empleo y el aumento de la desprotección del trabajo y el desplazamiento de un modelo de "ciudad abierta" a uno de "ciudad cerrada" (Svampa, 2001).(En página 98.)

12 Las "villas miseria" o "de emergencia", ganaron terreno por sobre otras modalidades de acceso a la tierra y vivienda para los sectores populares (Torres, 1992, 1993). Para muchos qom migrantes, el barrio de La Boca (en la ciudad de Buenos Aires), y particularmente Villa lapi (Partido de Quilmes) y Dock Sud (Partido de Avellaneda), se constituyeron en los lugares de primer asentamiento en la RMBA. (En página 98.)

13 Respecto de la duplicación de los asalariados precarizados - definidos como aquellos a quienes no se les realizan los descuentos jubilatorios-, Calvi y Benza (s/f) señalan que si se considera el conjunto de los asalariados, el porcentaje de puestos precarios se incrementó un $97 \%$, pasando del $22,5 \%$ en octubre de 1974 al $44,4 \%$ en mayo de 2003 . Si se descuentan los trabajadores del servicio doméstico, el incremento en la proporción de asalariados precarios, en ese mismo lapso, la cifra sería 114\% — pasando de un 19,0\% a un 40,7\%. (En página 98.)

14 La tasa de desocupación de dicho aglomerado, de acuerdo con Maceira (2012), creció entre el 6,3\% y el 16,4\% de la Población Económicamente Activa entre 1991 y el 2003, lo que implicó un aumento absoluto y relativo de esta mayor que en el resto de los aglomerados urbanos. El desempleo no solo se produjo por la incapacidad de absorción de nuevos trabajadores sino por la destrucción de los puestos de trabajo pleno. (En página 98.) 


\section{Referencias bibliográficas}

" Assies, W. (2009). Pueblos indígenas y sus demandas en los sistemas políticos. Revista CIDOB d'Afers Internacionals, 85, 89-107.

»Attías, A. M., y Lombardo, R. D. (2014). Población originaria de la Provincia del Chaco. Territorios, dominación y resistencias. Theomai, (30), 65-80.

" Bigot, M., Rodríguez, G. y Vázquez, H. (1991). Asentamientos Toba-qom en la ciudad de Rosario. Procesos étnicos identitarios. América Indígena, 51(1), 217-251.

" Bigot, M., Rodríguez, G. y Vázquez, H. (1992). Los asentamientos tobas en la ciudad de Rosario. En J. C. Radovich y A. O. Balazote (Comps.). La problemática indígena (pp. 81100). Buenos Aires: Centro Editor de América Latina.

» Bigot, M., Rodríguez, G. y Vázquez, H. (1995). Construcción de liderazgos y de estrategias etnopolíticas en grupos de familias tobas asentadas en la ciudad de Rosario. Papeles de Trabajo, 4, 11-21.

" Calvi, G. y Benza, G. (s/f). Precariedad laboral y distribución del ingreso en el Gran Buenos Aires (1974-2003). 7mo Congreso Nacional de Estudios del Trabajo. Recuperado de https://www.aset.org.ar/congresos/7/03005.pdf

"Cardini, L. A. (2006). Artesanías y pueblos originarios. Aproximaciones para su estudio en la ciudad de Rosario, Argentina. Runa, 26(1), 263-288.

"Carlino, A. M. (2001). El impacto de la globalización sobre el algodón en el Chaco. Indicadores Económicos, 46, 1-15. Recuperado de http://eco.unne.edu.ar/economia/revista/46/o2.pdf

"Castilla, M. I. (2017). De lo global a lo local: políticas públicas y surgimiento de organizaciones étnicas en Pampa del Indio, Chaco. Papeles de trabajo, 34, 29-44.

"De La Maza Cabrera, F. (2012). La noción de "indígena” en las prácticas estatales locales. El caso de tres comunas de la Araucanía, Chile. Cuadernos de Antropología Social, 83, 91-117.

» Díaz Polanco, H. (2006). El laberinto de la identidad. México DF: Universidad Nacional Autónoma de México.

"Domínguez, D. (2005). Movimiento campesino e indígena en Argentina: Luchas contra el saqueo y colonialismo del siglo XXI ¿Movimiento campesino en Argentina? Recuperado de http://vivalatinoamerica-vivalatinoamerica.blogspot.com/2008/11/movimiento-campesino-e-indigena-en.html

»Ebel, G. A. (2013). Crecimiento, distribución y composición de la población urbana y rural en el Chaco entre 1991 y 2010. ADNea, 1, 77-86.

» Garbulsky, E. (1994). Cuestión étnica-Cuestión social. Las fronteras contemporáneas de los grupos toba (qom). Rosario en el umbral del siglo XXI. Andes, Antropología e Historia, 6,393-420.

" García, I. L. (2007). Los cambios en el proceso de producción de algodón en el chaco en las últimas décadas y sus consecuencias en las condiciones de vida de minifundistas y trabajadores vinculados. Revista de Estudios Regionales y Mercado de Trabajo, 3, 111-134. Recuperado de http://www.simel.edu.ar/archivos/documentos/RS36\%20Garcia.pdf

» Giarracca, N. y Teubal, M. (2010). Disputas por los territorios y recursos naturales: el modelo extractivo. Revista ALASRU, 5, 113-133.

" Gordillo, G. (2006). En el Gran Chaco. Antropologías e historias. Buenos Aires: Prometeo. 
" Hale, C. (2002). Does multicultiralism menace? Governance, cultural rights and the politics of identity in Guatemala. Journal of Latinamerican Studies, 34(3), 485-524. doi. org/10.1017/So022216X02006521

» Harvey, D. (2007). Breve historia del neoliberalismo (Vol. 49). Madrid: Akal.

» Harvey, D. (2013). Ciudades rebeldes. Del derecho a la ciudad a la revolución urbana. Buenos Aires: Akal.

» Iñigo Carrera, V. (2012). Movilización indígena en el Chaco argentino. Acción y conciencia políticas entre los qom del este de Formosa. Indiana, 29, 273-301.

» Luna, D. H. (2016). Agronegocio y violencia: dinámicas locales de un modelo global. Revista Theomai, 34, 20-31.

» Maceira, V. (2012). Notas para una caracterización del Área Metropolitana de Buenos Aires. Boletín Informativo. Observatorio das Metropoles, 224, 1-9. Recuperado de http://www.cin.edu.ar/descargas/asuntosacademicos/8-07-\%20Propuesta\%2olng.\%20 SPada\%20SPU-\%2oPROYECTO\%20DE\%20MODIFICACION\%2ORESOLUCION\%20 $602 \% 20(2) . p d f$

" Maidana, C. (2011). Migrantes Toba (qom). Procesos de territorialización y construcción de identidades (Tesis de Doctorado). Universidad Nacional de La Plata, Facultad de Ciencias Naturales y Museo: La plata, Argentina.

» Manzano, V. (2013). La política en movimiento. Movilizaciones colectivas y políticas estatales en la vida del Gran Buenos Aires. Rosario: Protohistoria.

" Muñoz, R. y Gallo, S. A. (2012). Conflictos por la tierra: El caso de la Unión Campesina del Chaco, 2002-2011. VII Jornadas de Sociología de la UNLP, Universidad de La Plata, La Plata, Argentina.

»Pertile, V. C. (2004). Ampliación de la frontera agropecuaria chaqueña: El Oeste Chaqueño y el cultivo algodonero. Revista Geográfica Digital, 1, 1-15. Recuperado de http:// hum.unne.edu.ar/revistas/geoweb/Geo1/archivos/iighi.pdf

» Rosati, G. 2008. Un caso de crisis de la pequeña propiedad agrícola. Acercamiento empírico al movimiento de la estructura económica del campo chaqueño. PIMSA Documentos y Comunicaciones, 8-27. Recuperado de http://www.pimsa.secyt.gov.ar/publicaciones/DT\%2069.pdf

» Slutzy, D. (2005). Los conflictos por la tierra en el área de expansión agropecuaria del NOA con especial referencia a la situación de los pequeños productores y a los Pueblos Originarios. Revista Interdisciplinaria de Estudios Agrarios, 23, 59-100.

"Stavenhagen, R. (2002). Indigenous peoples and the state in Latin America: an ongoing debate. En R. Sieder (Ed.). Multiculturalism in Latin America. Indigenous Rights, diversity and democracy (pp. 24-44). Londres: Institute of Latin American Studies.

» Stoler, A. (1987): Transiciones en Sumatra: el capitalismo colonial y las teorías sobre la subsunción. Revista Internacional de Ciencias Sociales, 114, 103-125.

»Svampa, M. (2001). Los que ganaron. La vida en los countries y barrios privados. Buenos Aires: Biblos.

" Tamagno, L. (2001). Nam qom Hueta 'a Na dockshi Lma: Los tobas en la casa del hombre blanco. Identidad, memoria y utopía. La Plata: Ediciones al Margen.

» Tamagno, L. (2014). Indígenas en la ciudad. Organización política en contextos de tensión entre patrimonio cultural y políticas públicas. En L. Tamagno y M. Maffia (Coords.). Indígenas, africanos y afrodescendientes en la Argentina. Convergencias, divergencias y desafíos (pp. 43-62). Buenos Aires: Biblos. 
» Torres, H. (1992). Cambios en la estructura socioespacial de Buenos Aires a partir de la década de 1940. En J. Jorrat y R. Sautu (Comps.). Después de Germani. Exploraciones sobre estructura social Argentina (pp. 158-175). Buenos Aires: Paidós.

» Torres, H. (1993). El mapa social de Buenos Aires (1940-1990). Buenos Aires: Ediciones Fadu.

»Valenzuela, C. y Scavo, A. V. (2008). La trama territorial del algodón en el Chaco. Transformaciones recientes desde la perspectiva de los pequeños y medianos productores. IX Encuentro Nacional de la red de Economías Regionales en el marco del Plan Fénix. Conflictos y transformaciones del territorio. Procesos sociales del último medio siglo. Recuperado de http://www.econ.uba.ar/planfenix/economias_regionales/comision\%20D/o3-Valenzuela-Scavo\%204.pdf

»Vázquez, H. (2000). Procesos identitarios y exclusión sociocultural. La cuestión indígena en la Argentina. Buenos Aires: Biblos.

»Viglianchino, M. (1997a). Redes, cuasigrupos y articuladores: algunas reflexiones desde la problemática de las familias Tobas (qom) de Empalme Graneros. Papeles de Trabajo, 6, 171-183.

»Viglianchino, M. (1997b). Género y etnía: reflexiones sobre un grupo de mujeres tobas migrantes. V Congreso Argentino de Antropología Social, Universidad Nacional de La Plata. La Plata, Argentina. Recuperado de http://www.equiponaya.com.ar/congresos/contenido/laplata/LP1/12.htm

» Weiss, M. L., Engelman, J. y Valverde, S. (2013). Pueblos indígenas urbanos en Argentina: un estado de la cuestión. Revista Pilquén, 16, 1-15.

»Weiss, M. L. (2015). Políticas públicas, proceso organizativo y adscripción étnica en una comunidad indígena del conurbano Bonaerense. Papeles de Trabajo, 20, 1-15.

»Wright, P. (1999). Histories of Buenos Aires. En E. Miller (Ed.). Peoples of Gran Chaco (pp. 135-156). Londres: Bergin and Garvey.

»Zorzoli, F. (2016). Disputas territoriales en el centro-norte de la provincia de Chaco. Cuadernos de Antropología, 15, 9-26.

\section{Otras fuentes consultadas}

» Boletín Oficial de la República Argentina (2017). Segunda sección, Año CXXV, Número 33.686.

» Instituto Nacional de Estadistica y Censo (INDEC). (2001). Censo Nacional de Población, Hogares y Viviendas. Buenos Aires, Argentina.

»Instituto Nacional de Estadistica y Censo (INDEC). (2005). Encuesta complementaria de pueblos indígenas (2004-2005). Buenos Aires, Argentina.

»Instituto Nacional de Estadistica y Censo (INDEC). (2012). Censo Nacional de Población, Hogares y Viviendas 2010. Tomo 1. Buenos Aires, Argentina. 
